\title{
Advanced Dynamic Autonomous Knowledge Learning Method for Distance Learning
}

\author{
Yanfang Fu \\ School of Computer Science and Engineering, \\ Xi`an Technological University \\ Xi'an, 710032, China \\ e-mail: $149481698 @ q q . c o m$
}

Xing Li

School of Computer Science and Engineering,

$$
\begin{gathered}
\text { Xi`an Technological University } \\
\text { Xi'an, 710032, China } \\
\text { e-mail: 825338005@qq.com }
\end{gathered}
$$

\author{
Xueyao Feng \\ School of Computer Science and Engineering, \\ Xi an Technological University \\ Xi'an, 710032, China \\ e-mail: $903840457 @ q q . c o m$ \\ Jing Ma \\ School of Computer Science and Engineering, \\ Xi'an Technological University \\ Xi'an, 710032, China \\ e-mail: 410329074@qq.com
}

\begin{abstract}
As the manufacturing of vast amount of information transmits at an unprecedented rate, traditional learning is no way to meet the needs of open learning and lifelong learning, how can distance learning be used to help us study become a problem to think about .Distance learning requires improvement of interactivity and initiative to achieve their aptitude. Using a variety of formats data, learners master the initiative in the learning process, it is not easy to lead to deviation from the learning goals phenomenon. To this end, in order to solve this problem, we use the basis of ILTMK theory and distributed interactive technology to introduce the active learning factors and propose a dynamic autonomous knowledge learning model and build its model's simulation running environment and related mechanism. Through simulation experiment, we can verify the effectiveness of the autonomous learning methods.
\end{abstract}

Keywords-Distance Learning; ILTMK theory; Distributed Interactive Technology; Dynamic Autonomous Knowledge Learning Model

\section{INTRODUCTION}

Looking at the source of knowledge view, Knowledge is that the knower grasps the essence of things through the phenomenon. So, Bystanders knowledge view derived from the ancient Greek natural philosopher, which hold by Socrates and others, lead to the "acceptance learning" long period domination of human way of learning[1-2]. In China, participant knowledge view of Basic Education Reform is ' inquiry learning'. The history of education practice shows, only participant knowledge view instead of bystander knowledge view, inquiry learning may be instead of reception learning. Knowledge contains a personal factor. Knowledge acquisition process is the learners to participate in the process of knowledge construction. Jone Deway would explain that participant knowledge view is interaction of the human and the environment [3]. Inquiry learning not only focuses on participant personal experiences process, more emphasis on personal understanding [4].

With the popularity of Internet, distance learning has gone from a letter-based, based on broadcast television systems "receiving learning" toward network-based (Baidu library and Blackboard) "research study" direction. Distance learning is a complement to a new independent study model. Now some intelligent distance learning increases chat rooms, electronic whiteboard and virtual classroom, and other functions to improve the learning effect, but it is only to 
provide support in the learning environment, did not achieve the custom teaching mode of learner's active learning. Existing model commonly used model based on Agent technology or ILTMK (Intelligent Long-distance Teaching Model based on Knowledge)[5].However, at present, most distance learning model is a simple information-sharing individual learning styles. Just solved how to make use of the Internet technology, makes the geographical separation of some groups can share learning materials, learners are always in a passive learning mode. So far no one is satisfied with the distance learning model [6]. If the learners can interact with active learning, distance learning can produce good learning results.

As information technology, especially with the rapid development of network technology and communication technology, distance learning and creates a rapidly improve our knowledge [9]. How to establish interactive mode between the learner and knowledge grantee, plays an important role in research and innovation.

\section{RELEVANT THEORIES OF THE DISTANCE LEARNING}

To make full use of the knowledge resources on the network, we must adopt a deeper sharing strategy that absorbed in the knowledge sharing or knowledge unit sharing[7]. A knowledge unit or learning entity is relatively independent, and it stands for one element of the knowledge unit, knowledge can be hierarchical and nested, and itself is an independent description metadata. LO can be divided into two parts, namely the metadata and content data. Metadata section describes the characteristics of the LO, content data part gives the knowledge that represented by LO and the information. In order to realize the intelligent reasoning of the system, we use the production system to represent knowledge and information [8]. The reason why we choose this knowledge representation is that it has many advantages, especially its easy understanding, availabilities and manageability. Meanwhile, today's most intelligent application adopted this kind of knowledge representation. Production system contains a set of production(or production rules) and facts, production rules use IFTHEN to show the knowledge, "attribute/ value" pairs are used to means the facts. Production rules has the following form:

If $\quad(<$ conditon $1><$ condition $2><$ condition $3><$ condition $\mathrm{n}>$ )

Then

( $<$ conclusion $1><$ conclusion $2><$ conclusio $3>---<$ conclusion $\mathrm{n}>)$

When satisfy the application condition, the data will be operated by the order which the conclusion talks. The form of the LO in the production can be constants, variables, unit groups or tree, figure.

\section{LEARNING MODEL BAESD ON INTERACTOIN}

\section{A. Characteristics of active distance-learning model design}

Remote active learning model should have the following characteristic elements. (1)boundary and exclusivity, which means personnel composition in a learning subject is clear, whether to learn the subject or not needs to be identified;(2)the purpose, learning subject exists as a certain purpose, also as the learning interaction that sharing some knowledge.(3)Rules, each member must abide by certain interactive code of conduct; (4)the obligations, In the process of learning, each knowledge sender bears certain teaching responsibility;(5)Independence, that is ,every member can make choice about their own rights and awareness.

The concept of the "interaction" firstly appeared in communication and sociology, in sociology, the definition of the interaction is ways and processes of interactive influences, effects produced between humans and groups."Interactive" dynamically performed the person's social relations, all kinds of complicated social phenomenon are caused by interaction. When thinking about interaction in the field of communication[9], we find that the interaction's is more likely to focus on the flow and exchange of study information, or relationship among transmission elements.

Simple "teaching" or "learning" in the distance learning is difficult to reach the expected goals, the entire learning process should be placed in "relationship" and "interactive" 
so that it can be better achieved. Therefore, interaction stands out of the virtual learning community. In the learning process, we call the interaction produced from learners interaction with others through internet or with their teachers the interactive spread[10]. Due to the nature of distance education, the teachers and students are often in different location , and time and space are relatively separated in the whole education, Therefore, purposeful and bilateral will be very obvious in the transmission of teaching, and interaction will be more important in teaching. Not free interactive communication often makes it more difficult for learners to improve themselves.

So we can analyze that the process of the knowledge learning can be partitioned into several periodic learning interactive topic. Each topic still be divided into several learning content, send requests to knowledge giver, when satisfy the condition of subject and the synchronization time, knowledge giver can respectively receive the request signals of each learner and not confused. Meanwhile, knowledge which knowledge giver send to multiple learners are sequentially arranged in a specific time to teach, each learner just needs to received it within the specified time so that they can distinguish their intellectual theme from confused knowledge learning process and receive it down. In distance learning network, it is impossible for knowledge giver to master all the knowledge, he still needs to learn from the other knowledge giver in the virtual network, when two or more knowledge giver send knowledge data with same subject at the same time, or send data to same knowledge learner, then the conflict happens, so we should schedule the time of knowledge interaction.

\section{B. Autonomous learning measurement method}

We should give two definitions in facing with the knowledge interaction scheduling:

- In the network, each learner exists at least one interactive subject in a scheduling cycle.

- Network learners cannot receive knowledge and send new knowledge at the same time, and learners can't receive more than one of the knowledge interaction either.
Each learner is learners in certain knowledge subjects, However, sometimes they can be the knowledge giver, so it increases the complexity of the system modeling. Therefore, the problem of scheduling knowledge interaction is to assigned knowledge content to learning object according to different subjects[11]. According to the knowledge content's interest, we can be divide into two groups, that's learners and the grantors. Assuming that the entire network of interest have $\mathrm{A} 1, \mathrm{~A} 2 \ldots \mathrm{An}$, and their knowledge content is divide into $\mathrm{L} 1, \mathrm{~L} 2 \cdots \mathrm{Lm}$. We can see it as a graph $\mathrm{G}(\mathrm{V}, \mathrm{E})$, of which the node $\mathrm{V}(1,2,3$ and $\mathrm{n})$ stands for knowledge interest, $\mathrm{n}$ is for the number of interest, and the edge of $\mathrm{E}$ is for the collection of the interactions. When node $\mathrm{i}$ and $\mathrm{j}$ can interact with each other, we can think that there exists a undirected edge $e_{i, j}$ between two nodes, and $e_{i, j} \in E$, then the node $\mathrm{i}$, $\mathrm{j}$ is one jump neighbor same with the learners and the knowledge givers; if the node $\mathrm{i}, \mathrm{j}$ cannot directly communicate with each other, it means that they don't have the same content of the knowledge, then we need to find out the knowledge giver about this knowledge content, Therefore, only through an intermediate node $\mathrm{k}$, can it do the communication interaction, that is ,there exist e and e, so the node $\mathrm{i}, \mathrm{j}$ are two jumps neighbor node. It is an adjacency matrix. We can describe the network with an symmetric binary $\mathrm{n}$ n matrix, as:

$$
\begin{aligned}
C[G]=\left[C_{i j}\right]_{n \times n}(i, j=1,2 \ldots n) \\
\left(\mathrm{C}_{i j}=\left\{\begin{array}{c}
1 \text { node. } \mathrm{i}, \mathrm{j} . \text { have.edges } \\
0
\end{array}\right)\right.
\end{aligned}
$$

Another $n \times n$ matrix:

$$
\begin{gathered}
D(G)=\left[d_{i j}\right]_{m \times n}(i, j=1,2 \ldots n) \quad\left(\mathrm{D}_{i j}=\right. \\
\left\{\begin{array}{c}
1, \text { node. i j.is.two, jumps.neighbor } \\
0, \quad \text { else }
\end{array}\right)
\end{gathered}
$$

Considering above two jumps node can realize the spatial reuse, it can finally obtain the optimal autonomous learning model. The network has a number of $\mathrm{M}$ of the knowledge content,we can use a $\mathrm{M}^{\times} \mathrm{N}$ binary matrix $\mathrm{T}=$ $\left[t_{m i}\right]_{M \times N}$ 


$$
t_{m i}=\left\{\begin{array}{c}
1 \text { knowledgecontent.to.learner. } i \\
0 \\
\text { else }
\end{array}\right.
$$

Interactive rate:

$\rho_{i}=\frac{\text { knowledge.content.number.to.learner } . i}{\text { knowledge.topic.number }}=\frac{\sum_{m=1}^{M} t_{m i}}{M}$

The entire network's interactive rate is:

$$
\rho=\frac{1}{N} \sum_{i=1}^{N} \rho_{i}=\frac{1}{M N} \sum_{m=1}^{M} \sum_{i=1}^{N} t_{m i}
$$

By two limiting conditions, two learners can send the request of the knowledge data at the same time only on the condition that two learners are separated by more than two jumps, we summarized the above content as the following model:

$$
\text { Min M and Max } \rho
$$

Limiting conditions:

$$
\begin{aligned}
& \mathrm{C} 1: \sum_{m=1}^{M} t_{m i} \geq(i \in[1, N]) \\
& \mathrm{C} 2: c_{i j}+t_{m i}+t_{m j} \leq 2,(i, j \in[1, N], i \neq j, m \in[1, M]) \\
& \mathrm{C} 3:{ }^{c_{i k} t_{m i}+c_{j k} t_{m j} \leq 1,(i, j, k \in[1, N], i \neq j, j \neq k, k \neq i, m \in[1, M])}
\end{aligned}
$$

The main idea of the interactive process is: Interaction is that each learner interact with knowledge giver according to the fixed knowledge content point and the chronological order on condition that they have common learning subject. The learners active behavior allows them to get more study time. If there is no learning behavior to release the study time, then let the knowledge giver's interaction have a free time, if other learners initiative is more active and don't have the enough to study further, then he can dispute with free time and improve more.

\section{BUILD SIMULATION MODEL BASED ON THE OPNET}

Data Packages in OPNET consist of one or more fields, package format editor is used to define the package format, data packages in each fields has name, size(which length is bit), color, type and default values. Using graphical way, it can directly display the package's each fields, The size of the fields can exactly reflect the bit length in package. We designed three different types of data packages as three different types of the data grouping, that is:knowledge content data grouping, active application grouping and knowledge givers's reply grouping.

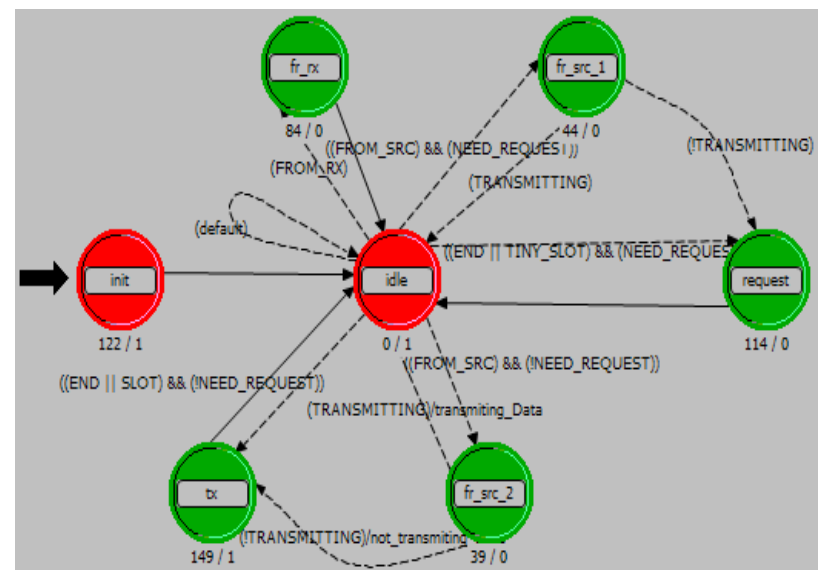

Figure 1. Learners process model

This process mainly includes the following states( fig.1), each state's function is introduced as follows:

- Init state: this state is mainly realized the initialization of the simulation data, initial object mainly includes: active interaction time slice, interactive time, number of the knowledge subject, number of the knowledge content etc. After completing the initialization of an object, call the interrupt function op_intrpt_schedule_self (op_sim_time (), 0), it will lead to process's state transformation from producing interruption at the current time to the request state only to do the intern's judgment.

- Idle state: free wait state, after all the state in performing the exit code should them return to this state, waiting for the right conditions for the next jump, In the exit code of this state will determine the current simulation's interruption type, and assign to variable current_intrpt_type, then determine whether produce the current node's interactive interruption or not, according to the type of the variable current_intrpt_type. If it is,then turned to the request or state tx to send the request package or data package. 
- $\quad$ Fr_rx state: responsible for identifying the type of the data packages that from the network receiver. Extract the corresponding information in the field ACK_Data,judge whether current node's interactive application is success or not, if yes, then extract the knowledge content's information that contains in the ACK_Data field of the data packages, modify the if_need_request to 0 , means application succeed. So there is no need to send the application grouping, then will cycle around in the state of idle, fr_rx, tx and fr_src_2, so the knowledge giver will send knowledge request in the assigned time slices until data queue is empty.

- Fr_src_1 state: The condition that transform from idle state to this state is that source module in the Nodes model generates a package, and has not yet been assigned the interactive time slice.

- Request state: The state of request is mainly responsible for calculating the current active application's time slice position, determine whether to enter their own interaction time, if he entered into his interaction time, then fill in the fields of information, send secondary intellectual content after set up the fields information; if not into its time slice or the current time slice has exceeded its clock, then compute the next arrival time(absolute simulation time ), and set up a break at this time.

- Fr_src_2 state: like the fr_src_1 status's function, fill in each field of the data package which receives from the data source, and insert it into the queue. The difference is that when the data source produces one package, only when the site's application for time slice succeed, can state transform from idle to fr_src_2.

- Tx state: when the node's application queue is not empty, sent data in this time slot. If the data grouping exists in the queue, you need to send the interaction request packet to the knowledge giver, apply for a number of time slice and use it to send data from a queue. Process at this time in idle, fr_rx, fr_src_1 and request jump between four states.
After the success of the application, each frame of data within the time slot waiting for their own time slot to send data. This process will be idle, fr_rx, fr_src_2 and tx , judge the time slice and send the data of internship for data to cycle until the system closed.

\section{SIMULATION EXPERIMENT}

The scale of the personnel simulation is about 480 people (Fig.2), the learners are set to the same request rate (intervalsetting function is negative expo-numberdistribution function),knowledge givers also bears for the same rate interact with learners. A learning subject forms one subnet, the learners interactwithknowledge giver to complete each content of the study, the learners may be as learners involved in the other subject learning subnet, a large number of this is nested.

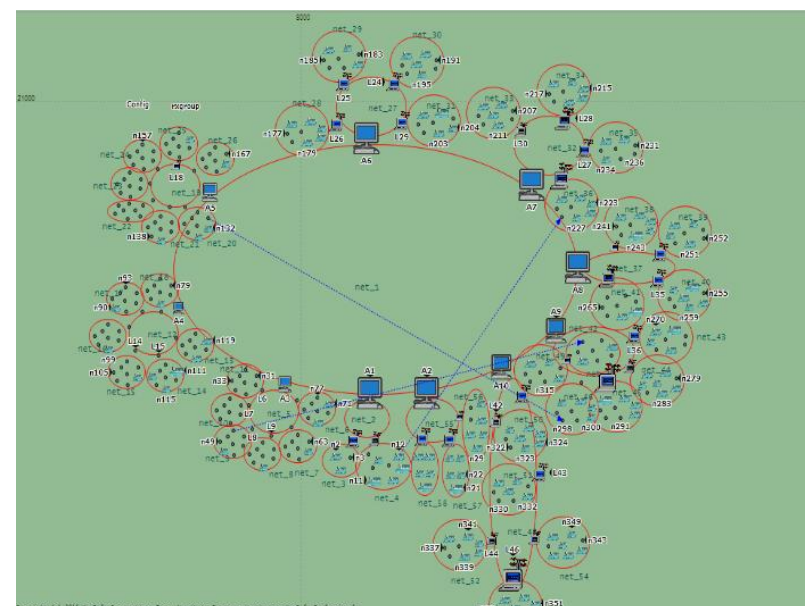

Figure 2. Modeling of simulated network level

We can see from the diagram that knowledge giver under the theme interact with a large number of students, and the learners complete the knowledge content learning. From the figure 3 , we can see that the improvement of the new model reflects the active learning behavior of learners. When the model adopting the dynamic autonomous learning, learners in sending interactive data clearly in dynamic changes (as shown in figure 4). Can be seen from the figure b, learners after receiving the grantor knowledge content, knowledge acquisition of related request change significantly, the interesting thing is the simulation data curve downward point is far lower than the original model of rate, this should 
be thatthe increase of the initiative will also reduce the interest in learning theme.

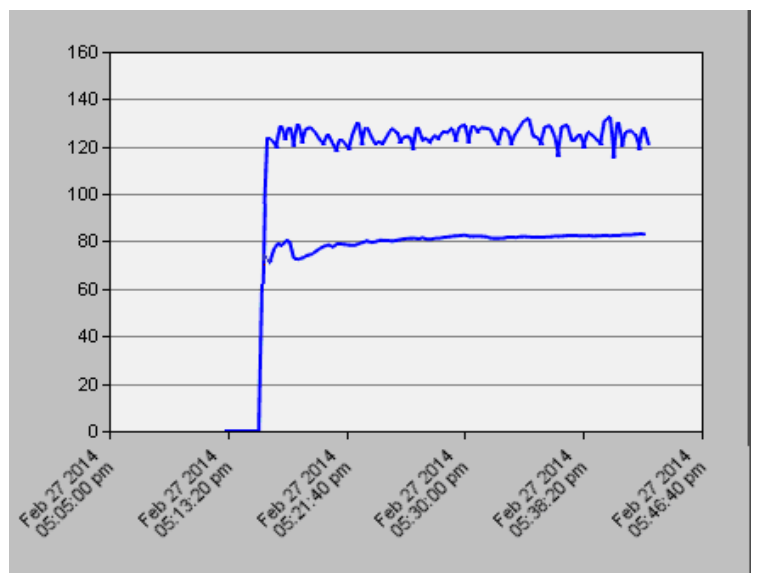

Figure 3. Learners average interactive rate

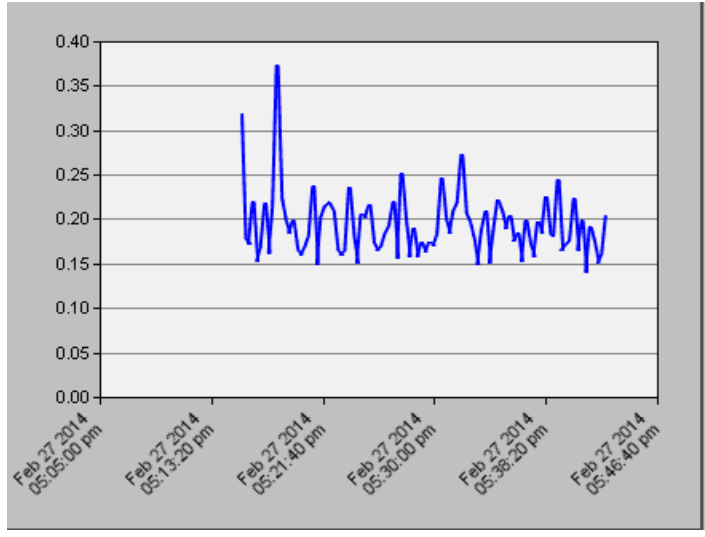

(a)Knowledge acquisition rate

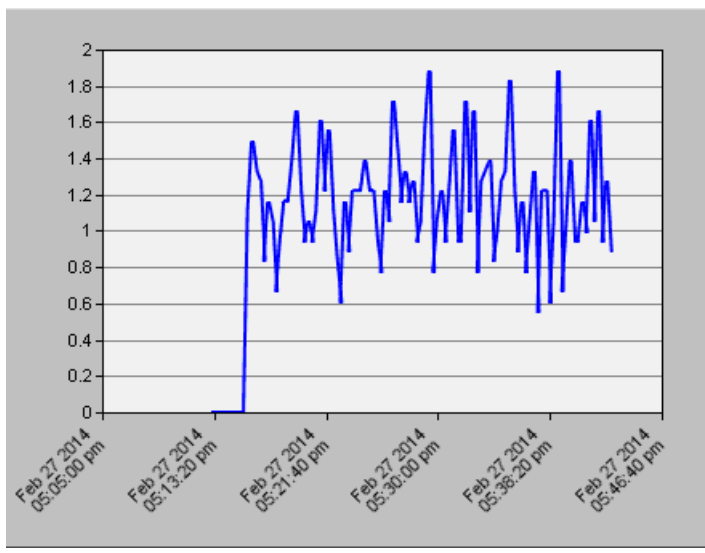

(b)Knowledge acquisition request rate

Figure 4. learners sending interactive data in dynamic changes
From the above two groups of simulation data, we can see that the improvement of the new model not only reflects the active learning behavior of learners, but also increase the degree to which are not interested in learning content.

\section{CONCLUSION}

The modern distance learning is bestowed with knowledge individuals, related to personal participation and learning knower. The enthusiasm of the individual, personal exploration, personal opinions are indispensable part of the knowledge. Knowledge itself is the intrinsic personal factors, the knowledge acquisition process is the knower individuals involved in the process of knowledge construction. Dewey explains the participants knowledge view as "interaction" of the human and the environment. Distance learning requires interactivity and initiative to improve, to achieve their aptitude, use now study in a variety of formats data, learners to master the initiative in the learning process, it is not easy to lead to deviation from the learning goals. To this end, on the basis of THLMT theory and distributed interactive technology, the introduction of active learning factors, put forward a kind of active learning rules of interaction, the interaction between learners and knowledge imparter is studied, under the condition of active learning, completely control rules generated by interactive knowledge subject data with method of study, established a model of autonomous learning. The model not only focus on learners "through" process, more emphasis on the learner in the experience, get personalized understanding in learning enthusiasm. This way of learning value is that learners in "facing the complex itself" keep some kind of "seek" uncertainty, in the process of "certainty for" construction "personal knowledge", in the process of "seeking" uncertainty "eagerly seeking knowledge", and will set up the learning process in the simulation software, in order to dynamically determine the distance learning program and related learning strategy, really improve the intelligent and personalized distance learning.

\section{ACKNOWLEDGMENT}

This research was supported by the industrial research project of Shaanxi science and technology department 
(nos.2016GY-088) and the educational reform fund of Xi'an Technological University (nos. 15JGZ05, 16JGY25, XAGDYJ160208).

\section{REFERENCES}

[1] Lee B, Yoon J,and Lee I, “Learners' acceptance of e-learning in South Korea: Theories and results," Computers \& Education. 53rd ed,vol.3, 2009,pp.1320-1329.

[2] Ahmed H M S. "Hybrid E-Learning Acceptance Model: Learner Perceptions," Decision Sciences the Journal of Innovative Education. 8th ed,vol.2, 2010,pp.313-346.

[3] Ikpeze $\mathrm{C} \mathrm{H}$,and Boyd $\mathrm{F}$ B. "Web-Based Inquiry Learning: Facilitating Thoughtful Literacy with WebQuests," Reading Teacher. 60th ed,vol.7, 2007,pp.644-654.

[4] Maudsley G, Williams E M I, and Taylor D C M. "Problem-based learning at the receiving end: A 'mixed methods' study of junior medical students' perspectives,"Advances in Health Sciences Education. 13th ed,vol.4, 2008,pp.435-451.
[5] Yun L X, sheng Z Y, and li Z S. "Intelligent Internet long-distance teaching model based on knowledge,"Journal of Hefei University of Technology. 2003,pp.514-519.

[6] English T, Harrison A L, and Hart A L. "A Distance Learning Model in a Physical Therapy Curriculum," Journal of Allied Health.1998, pp. 27.

[7] Jenkins J R P C G J M. "Special education and the regular education initiative: basic assumptions," Exceptional Children. 56th ed,vol.6, 1990,pp.479-491.

[8] Harjumaa M, and Oinas-Kukkonen H. "TowardsDeeper Understanding of Persuasion in Software and Information Systems," International Conference on Advances in Computer-human Interaction. IEEE Computer Society, 2008, pp.200-205.

[9] Gellman-Danley B, and Fetzner M J. "Asking the really tough questions: Policy issues for distance learning." Online Journal of Distance Learning Administration . 1998,pp.49-54

[10] Qing P, Yun X, and Shou-hong W."Research and Implementation of Animation Technology on Distance Teaching Courseware," Application Research of Computers. 21st ed,vol.2, 2004,pp.144-146.

[11] Boyle T. "Design principles for authoring dynamic reusable learning objects," Australian Journal of Educational Technology. 19th ed,vol.1, 2003,pp.46--58. 